\title{
Scheduling English Football Fixtures: Consideration of Two Conflicting Objectives
}

\author{
Graham Kendall • Barry McCollum • \\ Frederico Cruz • Paul McMullan
}

\begin{abstract}
In previous work the distance travelled by UK football clubs, and their supporters, over the Christmas/New Year period was minimised. This is important as it is not only a holiday season but, often, there is bad weather at this time of the year. Whilst searching for good quality solutions for this problem, various constraints have to be respected. One of these relates to clashes, which measures how many paired teams play at home on the same day. Whilst the supporters have an interest in minimising the distance they travel, the police also have an interest in having as few pair clashes as possible. This is due to the fact that these fixtures are more expensive, and difficult, to police. However, these two objectives (minimise distance and minimise pair clashes) conflict with one another in that a decrease in one intuitively leads to an increase in the other. This paper explores this question and shows that there are compromise solutions which allow fewer pair clashes but does not statistically increase the distance travelled. This paper provides a more comprehensive study of the initial results presented at the previous PATAT conference. We present a more detailed set of computational experiments, along with a greater number of datasets. We conclude that it is sometimes possible to reduce the number of pair clashes whilst not significantly increasing the overall distance that is travelled.
\end{abstract}

Keywords Sport · Football $\cdot$ Scheduling $\cdot$ Multiobjective

Graham Kendall

School of Computer Science, University of Nottingham, NG8 1BB, UK

Tel.: +44 (0) 1158466514

Fax: +44(0) 1159514254

E-mail: gxk@cs.nott.ac.uk

Barry McCollum

School of Electronics, Electrical Engineering and Computer Science, Queen's University Belfast, BT7 1NN, UK

Frederico Cruz

Departamento de Estatstica - ICEx - UFMG, Av. Antnio Carlos, 6627, 31270-901 - Belo Horizonte - MG, Brazil

Paul McMullan

School of Electronics, Electrical Engineering and Computer Science, Queen's University Belfast, BT7 1NN, UK 


\section{Introduction}

The English Premier League is one of the most high profile, and successful, football (soccer in the USA) leagues in the world. It comprises 20 teams which have to play each other both home and away (i.e. a double round robin tournament), resulting in 380 fixtures that have to be scheduled. The other three main divisions in England (the Championship, League One and League Two) each have 24 teams, resulting in 552 fixtures having to be scheduled for each division. Therefore, for the four main divisions in England 2036 fixtures have to be scheduled every season. The divisions operate a system of promotion and relegation such that the teams in each division changes each year so it is not possible to simply use the same schedule every time.

Of particular interest are the schedules that need to be generated for the Christmas/New Year period. At this time of the year it is a requirement that every team plays two fixtures, one on Boxing Day (26th December) and one on New Years Day (1st January). Whilst scheduling these two sets of fixtures the overriding aim is to minimise the total distance that has to be travelled by the supporters. An analysis of the fixtures that were actually used, and also following discussions with the football authorities, confirm that this is a real world requirement and that the distances travelled by the supporters are the minimum when compared against other fixtures when all teams play. In addition, there are various other constraints that have to be respected, which are described in sections 3 and 4 .

The problem we tackle in this paper is to attempt to minimise two competing objectives to ascertain if there is a good trade off between them. The objectives we minimise are the distances travelled by the supporters and the number of pair clashes. Pairing matches two (or more) teams and dictates that these clubs should not play at home on the same day. If they do, this is termed a pair clash. In fact, a certain number of pair clashes are allowed. The exact number is taken from the number that were present in the published fixtures for a given season. Importantly, paired teams cannot play each other on the two days in question. This is treated as a hard constraint. It is this constraint that causes a problem. If we allow Liverpool and Everton (for example) to play each other, one set of supporters would only travel four miles. If these teams are paired (as they are) then they cannot play each other so the distances are likely to increase as either Liverpool or Everton would have to travel more than four miles. As pair clashes usually involve teams which are geographically close this gives rise to the conflicting objectives.

In [19], an initial study of the problem considered the 2003-2004 football season, suggesting that it may be possible to minimise both of these competing objectives but still produce results which are acceptable to both the supporters (who are interested in minimising the amount they travel) and the police (who are interested in having fewer pair clashes). In this paper, we carry out a more in depth study by considering more seasons and carrying out statistical analysis of the results in order to draw stronger conclusions.

\section{Related Work}

Producing a double round robin tournament is relatively easy in that the algorithms are well known, with the polygon construction method being amongst the most popular [9]. The problem with utlising such an algorithm is that the fixtures it generates, 
although being a valid round robin tournament, will not adhere to all the additional constraints for a particular problem. Moreover, every problem instance will be subtly different and, often, a bespoke algorithm is required for each instance. This is even the case when faced with seemingly the same problem. For example, the English Football League consists of four divisions and 92 teams. It would be easy to assume that once an algorithm has been developed it can be used every season. This may indeed be the case but due to the promotion/relegation system the problem changes year on year and, perhaps, there are additional features/constraints in one season that were not previously present. Rasmussen and Trick [21] provide an excellent overview of the issues, methods and theoretical results for scheduling round robin tournaments.

The Travelling Tournament Problem (TTP) [11] is probably the most widely used test bed in sports scheduling. The problem was inspired by work carried out for Major League Baseball [11]. The aim of the TTP is to generate a double round robin tournament, while minimising the overall distance travelled by all teams. Unlike the problem studied in this paper, it is possible to minmise the overall travel distance as teams go on road trips so, with a suitable schedule, the length of these trips can be reduced. The TTP is further complicated by the introduction of two constraints. The first says that no team can play more than three consecutive home or away games. The second stipulates that if team $i$ plays team $j$ in round, $r$, then team $j$ cannot play team $i$ in round $r+1$. These constraints add sufficient complexity to the problem so as to make it challenging, but it still does not reflect all the constraints that are present in the real world problem.

The TTP has received significant research attention. Some of the important papers being $[12,2,8,22,25]$. A recent annotated bibliography of TTP papers can be found in [18]. An up to date list of the best known solutions, as well as details of all the instances, can be found at the web site maintained by Michael Trick [23].

With respect to minimising travel costs/distances, previous studies have considered a variety of sports. Campbell and Chen [6] and Ball and Webster [3] both studied basketball, attempting to minimise the distance travelled. Bean and Birge [4] also studied basketball, attempting to minimise airline travel costs. Minimising travel costs was also the focus of [5], for baseball. Minimising travel distances for hockey [16] and umpires for baseball [15] have also been studied. Wright [28], as one part of the evaluation function, considered travel between fixtures for English cricket clubs. Costa [7] considered the National Hockey League, where minimisation of the distance travelled by the teams was just one factor in the objective function.

Urrutia and Ribeiro [24] have shown that minimising distance and maximising breaks (two consecutive home games (home break) or two consecutive away games (away break)) is equivalent. This followed previous work by de Werra [26,27] and Elf et al. [14] who showed how to construct schedules with the minimum number of breaks.

The scheduling problem that we are considering in this paper is minimising the distance travelled for two complete fixtures (a complete fixture is defined as a set of fixtures when every team plays) while, at the same time, minimising the number of pair clashes. These two complete fixtures can then be used over the Christmas holiday period when, for a variety of reasons, teams wish to limit the amount of travelling undertaken. Note, that this is a different problem to the Travelling Tournament Problem as the TTP assumes that teams go on road trips, and so the total distance travelled over a season can be minimised. In English football, there is no concept of road trips. Therefore, over the course of a season, the distance cannot be minimised. However, we can minimise the distance on particular days. Kendall [17] adopted a two-phase approach to produce two 
complete fixtures for this problem. A depth first search was used to produce fixtures for one day, for each division. A further depth first search created another set of fixtures for the second day. This process produced eight separate fixtures (two sets of fixtures for each division) which adhered to some of the constraints (e.g. a team plays at home on one day and away on the other) but had not yet addressed the constraints with regards to pair clashes (see [17] for a detailed description). The fixture lists from the depth first searches were input to a local search procedure which aimed to satisfy the remaining constraints, whilst attempting to minimise the overall distance travelled. The output of the local search, and a post-process operation to ensure feasibility, produced the results presented in the paper.

Overviews of sports scheduling can be found in $[13,9,10,21,29,20,18]$.

\section{Problem Definition}

In previous work [17] the only objective was to minimise the total distance travelled by the teams/supporters. The aim of that study was to investigate if we were able to generate better quality solutions than those used by the football league. We demonstrated that it was possible. As stated in the Introduction, the police also have an interest in the fixtures that are played at this time of the year. If we are able to generate acceptable schedules, with fewer pair clashes then the policing costs would be reduced.

The purpose of this paper is to investigate if there is an acceptable trade off between the minimisation of distance and the minimisation of pair clashes. In order to do this we will utilise a multi-objective methodology.

\section{Experimental Setup}

We use a two stage algorithm. In [17] a depth first search (DFS) was used, followed by a local search. DFS was used as we wanted to carry out a preliminary study just to see if this area was worthy of further study. As we were able to produce superior solutions to the published fixtures we have now decided to utilise more sophisticated methods, due to the large execution times of DFS which were typically a few hours for each division. In this work we utilise CPLEX as a replacement for DFS and simulated annealing [1] as a replacement for the local search. This reduces the overall execution time from tens of hours to a few minutes.

\subsection{Phase 1: CPLEX}

The first phase uses CPLEX to produce an optimal solution to a relaxed version of the problem. In generating relaxed optimal solutions we respect the following constraints, whilst minimizing the overall distance.

1. Each of the 92 teams has to play on two separate days (i.e. 46 fixtures will be scheduled on each day).

2. Each team has to play at home on one day and away on the other.

3. Teams are not allowed to play each other on both days.

4. A team is not allowed to play itself. 
The CPLEX model is executed four times. Each run returns the Boxing Day and New Years Day fixtures for a particular division. Each run takes less than 10 seconds.

In solving the CPLEX model we do not take into account many of the constraints that ultimately have to be respected. For example, pair clashes, geographical constraints such as the number of London or Manchester clubs playing at home on the same day etc. (see [17] for details).

\subsection{Phase 2: Simulated Annealing}

The schedules from CPLEX are input to the second phase, where we utilise simulated annealing. This operates across all the divisions in order to resolve any hard constraint violations whilst still attempting to minimise the distance.

The simulated annealing parameters are as follows:

Start_Temperature $=\mathbf{1 0 0 0}$ The same value is used across all seven datasets and was found by experimentation. We could have used different values for each dataset but we felt that it was beneficial to be consistent across all the datasets.

Stop_Temperature The algorithm continues while the temperature is $>0.1$.

Cooling Schedule CurTemp $=$ CurTemp ${ }^{*} 0.95$.

Number of Iterations 2000 iterations are carried out at each temperature.

\subsection{Evaluation Function}

The evaluation function we use for simulated annealing is dynamic in that the hard constraint violations are more heavily penalised as the search progresses. This enables more exploration at the start of the search, which gets tighter as the temperature is reduced. The objective function is formulated as follows:

$$
f(x)=d_{-} f b+d_{-} f y+w \times \text { penalty }
$$

where:

$d_{-} f b=$ total distance travelled by teams on Boxing Day.

$d_{-} f y=$ total distance travelled by teams on New Years Day.

$w=$ is a weight for the penalty (see below). It is given by (Start_Temperature -

CurTemp). Start_Temperature is the maximum temperature for the simulated annealing algorithm. CurTemp is the current temperature of the simulated annealing algorithm. As the simulated annealing algorithm progresses, the weight of the penalty gradually increases, driving the search towards feasible solutions, but allowing it to search the infeasible region at the start of the search.

penalty $=$ This is given by a summation of the following terms (the limits referred to are available in [17] and represent the values found by analyzing the published fixtures):

ReverseFixtures The number of reverse fixtures (the same teams cannot meet on both days).

Boxing Day Local Derby Clashes The number of paired teams playing each other on Boxing Day. 
New Years Day Local Derby Clashes The number of paired teams playing each other on New Years Day.

Boxing Day London Clashes The number of London clubs playing at home on Boxing Day, which exceed a given limit.

New Years Day London Clashes The number of London clubs playing at home on New Years Day, which exceed a given limit.

Boxing Day Greater Manchester Clashes The number of Greater Manchester based clubs playing at home on Boxing Day, which exceed a given limit.

New Years Day Greater Manchester Clashes The number of Greater Manchester based clubs playing at home on New Years Day, which exceed a given limit.

Boxing Day London Premier Clashes The number of Premiership London clubs playing at home on Boxing Day, which exceed a given limit.

New Years Day London Premier Clashes The number of Premiership London clubs playing at home on New Years Day, which exceed a given limit.

Boxing Day Clashes The number of Boxing Day clashes greater than an allowable limit.

New Years Day Clashes The number of New Years Day clashes greater than an allowable limit.

\subsection{Perturbation Operators}

Simulated annealing often has a single neighborhood operator but we have defined sixteen operators in order to match the hard constraints within the model. The operators are as follows:

1. Examines the Boxing Day fixtures and if the number of clashes exceeds an upper limit, randomly select one of the clashing fixtures and swap the home and away teams.

2. Same as 1 expect that it considers New Years Day fixtures.

3. Examines the Boxing Day fixtures and if the number of London based clubs exceeds an upper limit, randomly select one of the fixtures that has a London based club playing at home and swap the home and away teams.

4. Same as 3 except that it considers Greater Manchester based clubs.

5. Same as 3 except that it considers London based premiership clubs.

6. Same as 3 except that it considers the New Years Day fixtures.

7. Same as 4 except that it considers the New Years Day fixtures.

8. Same as 5 except that it considers the New Years Day fixtures.

9. Examines the Boxing Day and New Years Day fixture lists, returning the number of reverse fixtures (where team $i$ plays team $j$ and team $j$ plays team $i$ ). While there are reverse fixtures, one of the reverse fixtures on Boxing Day is chosen and the home team is swapped with a randomly selected home team, with the condition that the swaps must be made between teams in the same division. This operator iterates until all reverse fixtures have been removed from the fixture list.

10. Same as 9 except the swaps are made in the New Years Day fixtures.

11. This operator examines the Boxing Day and New Years Day fixture lists, returning the number fixtures where paired teams are playing each other. While this is the case, one of the Boxing Day fixtures is chosen and the home team is swapped with a randomly selected home team in the Boxing Day fixtures, with the condition that 
the swaps must be made between teams in the same division. This operator iterates until all local pair clashes have been removed from the fixture lists.

12. Same as 11 except the swaps are made in the New Years Day fixtures.

13. This operator chooses a random fixture from a candidate list (we use a candidate list size of 250) which represents the potential fixtures that have the shortest distances. Swaps are carried out in the Boxing Day fixtures in order to allow the two teams from the selected item in the candidate list to play each other. The necessary swaps are also done in the New Years Day fixture to ensure feasibility.

14. Same as 13 except that it considers the New Years Day fixtures.

15. Selects a random fixture in the Boxing Day fixture list and swaps the home and away teams.

16. Same as 15 , but swaps a random fixture in the New Years Day fixture list.

At each iteration, one of the sixteen operators is chosen at random. Start_Temperature is initially set to enable infeasible solutions during the early stages of the algorithm, but they are more heavily penalised at lower temperatures (eq. 1), ensuring that the final solution is feasible.

\subsection{Experimental Methodology}

We are investigating this problem from a multi-objective perspective but rather than using a multi-objective algorithm we run the same algorithm a number of times, adjusting the parameters for each run. As an example, for the 2002-2003 season the number of pair clashes, in the published fixtures, was 10 and 8 for Boxing Day and New Years Day respectively. We denote this as 10-8 in the tables below. Therefore, the first experiment fixes the values as 10 and 8 as the number of pair clashes that cannot be exceeded. In this respect, these values represent hard constraints. The next experiment reduces one of these values so that the next experiment uses $10-6$. We then reduce the other value to run a further experiment using 8-8. There are two points worthy of note. Firstly, we reduce the value by two as a pair clash of, say, Everton and Liverpool actually counts as two pair clashes as both teams are considered to be clashing. Secondly, we do not reduce the total number of pair clashes below 16 .

\section{Results}

Tables 1 thru 7 shows the results of each of the seven seasons that we use. The Clashes column shows the number of pair clashes (see section 4.5 for the notation that we use). Min represents the best solution found. Max is worst solution found and Average and Std Dev are self-explanatory. All experiments were runs 30 times.

Table 1 2002-2003: Summary of results from 30 runs

\begin{tabular}{lllll}
\hline Clashes & Min & Max & Average & Std Dev \\
\hline $10-8$ & 5243 & 6786 & 5630 & 288.46 \\
$10-6$ & 5674 & 7222 & 6183 & 410.71 \\
$8-8$ & 5562 & 6797 & 6070 & 309.50 \\
\hline
\end{tabular}


Table 2 2003-2004: Summary of results from 30 runs

\begin{tabular}{lllll}
\hline Clashes & Min & Max & Average & Std Dev \\
\hline $8-14$ & 5464 & 6173 & 5698 & 165.46 \\
$8-12$ & 5412 & 6519 & 5827 & 228.66 \\
$8-10$ & 5511 & 7093 & 6053 & 417.00 \\
$8-8$ & 5887 & 7674 & 6535 & 433.83 \\
$6-14$ & 5550 & 6334 & 5805 & 176.02 \\
$6-12$ & 5559 & 6587 & 6036 & 289.75 \\
$6-10$ & 5898 & 7416 & 6454 & 395.37 \\
$4-14$ & 5592 & 6911 & 6059 & 274.61 \\
$4-12$ & 5886 & 7848 & 6635 & 484.59 \\
$2-14$ & 6028 & 7704 & 6704 & 448.87 \\
\hline
\end{tabular}

Table 3 2004-2005: Summary of results from 30 runs

\begin{tabular}{lllll}
\hline Clashes & Min & Max & Average & Std Dev \\
\hline $10-10$ & 5365 & 6986 & 5644 & 318.33 \\
$10-8$ & 5345 & 6348 & 5727 & 259.17 \\
$10-6$ & 5812 & 7714 & 6431 & 421.63 \\
$8-10$ & 5443 & 6982 & 5923 & 469.01 \\
$8-8$ & 5645 & 7612 & 6428 & 550.67 \\
$6-10$ & 5810 & 7824 & 6486 & 487.26 \\
\hline
\end{tabular}

Table 4 2005-2006: Summary of results from 30 runs

\begin{tabular}{lllll}
\hline Clashes & Min & Max & Average & Std Dev \\
\hline $12-14$ & 5234 & 6046 & 5575 & 184.74 \\
$12-12$ & 5335 & 6002 & 5596 & 153.90 \\
$12-10$ & 5240 & 6511 & 5641 & 238.58 \\
$12-8$ & 5334 & 6423 & 5754 & 231.81 \\
$12-6$ & 5481 & 6958 & 6010 & 339.63 \\
$12-4$ & 6041 & 6989 & 6468 & 271.99 \\
$10-14$ & 5171 & 6683 & 5606 & 304.33 \\
$10-12$ & 5308 & 6322 & 5610 & 204.96 \\
$10-10$ & 5460 & 6674 & 5846 & 359.65 \\
$10-8$ & 5595 & 6380 & 5872 & 216.82 \\
$10-6$ & 6027 & 7561 & 6660 & 421.25 \\
$8-14$ & 5335 & 6674 & 5680 & 286.00 \\
$8-12$ & 5334 & 6133 & 5722 & 211.02 \\
$8-10$ & 5608 & 7078 & 5979 & 356.15 \\
$8-8$ & 6146 & 7277 & 6587 & 302.48 \\
$6-14$ & 5500 & 6694 & 5843 & 254.23 \\
$6-12$ & 5528 & 6655 & 5951 & 233.54 \\
$6-10$ & 5884 & 7291 & 6529 & 382.80 \\
$4-14$ & 5713 & 7391 & 6161 & 331.25 \\
$4-12$ & 6032 & 7904 & 6662 & 434.72 \\
$2-14$ & 6084 & 7551 & 6682 & 399.34 \\
\hline
\end{tabular}

In tables 8 and 9 we analyse the results from table 1 . Table 8 shows the results of independent two-tailed $t$-tests (at the $95 \%$ confidence level) to compare the means of each experiment against every other experiment for that season. Where two experiments are statistically significant the relevant cell shows "Yes", otherwise the cell is 
Table 5 2006-2007: Summary of results from 30 runs

\begin{tabular}{lllll}
\hline Clashes & Min & Max & Average & Std Dev \\
\hline $14-8$ & 5713 & 7040 & 6077 & 300.71 \\
$14-6$ & 5735 & 7065 & 6117 & 270.59 \\
$14-4$ & 5872 & 7000 & 6259 & 227.84 \\
$14-2$ & 6110 & 7778 & 6741 & 402.35 \\
$12-8$ & 5721 & 6784 & 6084 & 244.28 \\
$12-6$ & 5714 & 6894 & 6234 & 326.99 \\
$12-4$ & 6195 & 7546 & 6791 & 405.86 \\
$10-8$ & 5762 & 7671 & 6209 & 411.02 \\
$10-6$ & 5894 & 7376 & 6618 & 423.94 \\
$8-8$ & 6071 & 6958 & 6513 & 251.33 \\
\hline
\end{tabular}

Table 6 2007-2008: Summary of results from 30 runs

\begin{tabular}{lllll}
\hline Clashes & Min & Max & Average & Std Dev \\
\hline $14-10$ & 5366 & 5902 & 5595 & 145.26 \\
$14-8$ & 5403 & 5975 & 5674 & 152.93 \\
$14-6$ & 5425 & 7172 & 5870 & 372.17 \\
$14-4$ & 5690 & 6995 & 6172 & 364.78 \\
$14-2$ & 5905 & 7856 & 6698 & 435.98 \\
$12-10$ & 5370 & 6506 & 5736 & 294.88 \\
$12-8$ & 5321 & 7139 & 5850 & 338.15 \\
$12-6$ & 5625 & 7394 & 6084 & 365.93 \\
$12-4$ & 5961 & 7580 & 6575 & 411.41 \\
$10-10$ & 5340 & 6552 & 5754 & 228.71 \\
$10-8$ & 5616 & 6365 & 5944 & 183.52 \\
$10-6$ & 6101 & 7468 & 6619 & 369.10 \\
$8-10$ & 5536 & 7081 & 6056 & 369.47 \\
$8-8$ & 6091 & 7884 & 6725 & 402.08 \\
$6-10$ & 5951 & 7709 & 6647 & 381.12 \\
\hline
\end{tabular}

Table 7 2008-2009: Summary of results from 30 runs

\begin{tabular}{lllll}
\hline Clashes & Min & Max & Average & Std Dev \\
\hline $10-10$ & 5564 & 6806 & 5833 & 246.11 \\
$10-8$ & 5574 & 6235 & 5829 & 140.52 \\
$10-6$ & 5736 & 6523 & 6106 & 208.78 \\
$8-10$ & 5581 & 6817 & 5936 & 281.83 \\
$8-8$ & 5790 & 6900 & 6148 & 230.42 \\
$6-10$ & 5809 & 7194 & 6208 & 274.67 \\
\hline
\end{tabular}

empty. As an example, if we compare 10-8 (column) with 10-6 (row) in table 8 we see that the means (i.e. the travel distances from 30 independent runs) are statistically different. By comparing the means in table 1, 5630 and 6183 respectively, we conclude that reducing the number of pair clashes from 18 (10-8) to 16 (8-8) the travel distances for the clubs/supporters increases by a significant amount. Looking at 10-6 and 8-8, there is no statistical difference. However, as both of these experiments represent 16 pair clashes it is, perhaps, not surprising that the average distance travelled over the 30 runs is (statistically) the same. 
Table 9 summarises the results from table 8 by only showing those experiments where there are statistical differences, AND when the total number of pair clashes is different (i.e. it will ignore 10-6 and 8-8). We can see from table 9 that there are no experiments where we can reduce the number of pair clashes that leads to no statistical difference in the distance travelled.

Tables 10 and 11 show similar analysis for the 2003-204 season. Again, it is not possible to reduce the number of pair clashes without an (statistically) increase in the distance travelled.

Tables 12 and 13 are more interesting. Table 12 shows that there is no statistical difference between the 10-10 (20 pair clashes) experiment and the 10-8 (18 pair clashes) experiment. Removing all the noise from the table (see table 13) we can see that it is possible to reduce the number of pair clashes from 20 to 18 without a significant rise in the distance travelled (the respective means from table 3 are 5644 and 5727).

For the remaining four seasons, we only present the summary tables. Where a "Yes" appears in these tables (tables 14 thru 17) it indicates that it is possible to reduce the number of pair clashes and not have an (statistical) increase in travel distance. The tables show that there are a number of opportunities to reduce policing costs. We are probably most interested in the top rows as they represent the fixtures that were actually used.

Table 8 2002-2003: Are the Results Statistically Different?

\begin{tabular}{llll}
\hline Clashes & $10-8$ & $10-6$ & $8-8$ \\
\hline $10-8$ & $\mathrm{X}$ & Yes & Yes \\
$10-6$ & & $\mathrm{X}$ & $\mathrm{X}$ \\
$8-8$ & & & \\
\hline
\end{tabular}

Table 9 2002-2003: Are different total clashes significantly different?

\begin{tabular}{llll}
\hline Clashes & $10-8$ & $10-6$ & $8-8$ \\
\hline $10-8$ & $\mathrm{X}$ & & \\
$10-6$ & & $\mathrm{X}$ & \\
$8-8$ & & & $\mathrm{X}$ \\
\hline
\end{tabular}


Table 10 2003-2004: Are the Results Statistically Different?

\begin{tabular}{lllllllllll}
\hline Clashes & $8-14$ & $8-12$ & $8-10$ & $8-8$ & $6-14$ & $6-12$ & $6-10$ & $4-14$ & $4-12$ & $2-14$ \\
\hline $8-14$ & $\mathrm{X}$ & Yes & Yes & Yes & Yes & Yes & Yes & Yes & Yes & Yes \\
$8-12$ & & $\mathrm{X}$ & Yes & Yes & & Yes & Yes & Yes & Yes & Yes \\
$8-10$ & & & $\mathrm{X}$ & Yes & Yes & & Yes & & Yes & Yes \\
$8-8$ & & & & $\mathrm{X}$ & Yes & Yes & & Yes & & \\
$6-14$ & & & & & $\mathrm{X}$ & Yes & Yes & Yes & Yes & Yes \\
$6-12$ & & & & & & $\mathrm{X}$ & Yes & & Yes & Yes \\
$6-10$ & & & & & & & $\mathrm{X}$ & Yes & & Yes \\
$4-14$ & & & & & & & & $\mathrm{X}$ & Yes & Yes \\
$4-12$ & & & & & & & & & $\mathrm{X}$ & \\
$2-14$ & & & & & & & & & & $\mathrm{X}$ \\
\hline
\end{tabular}

Table 11 2003-2004: Are different total clashes significantly different?

\begin{tabular}{lllllllllll}
\hline Clashes & $8-14$ & $8-12$ & $8-10$ & $8-8$ & $6-14$ & $6-12$ & $6-10$ & $4-14$ & $4-12$ & $2-14$ \\
\hline $8-14$ & $\mathrm{X}$ & & & & & & & & & \\
$8-12$ & & $\mathrm{X}$ & & & & & & & & \\
$8-10$ & & & $\mathrm{X}$ & & & & & & & \\
$8-8$ & & & & $\mathrm{X}$ & & & & & & \\
$6-14$ & & & & & $\mathrm{X}$ & & & & & \\
$6-12$ & & & & & & $\mathrm{X}$ & & & & \\
$6-10$ & & & & & & & $\mathrm{X}$ & & & \\
$4-14$ & & & & & & & & $\mathrm{X}$ & & \\
$4-12$ & & & & & & & & & $\mathrm{X}$ & \\
$2-14$ & & & & & & & & & & $\mathrm{X}$ \\
\hline
\end{tabular}

Table 12 2004-2005: Are the Results Statistically Different?

\begin{tabular}{lllllll}
\hline Clashes & $10-10$ & $10-8$ & $10-6$ & $8-10$ & $8-8$ & $6-10$ \\
\hline $10-10$ & $\mathrm{X}$ & & Yes & Yes & Yes & Yes \\
$10-8$ & & $\mathrm{X}$ & Yes & & Yes & Yes \\
$10-6$ & & & $\mathrm{X}$ & Yes & & \\
$8-10$ & & & & $\mathrm{X}$ & Yes & Yes \\
$8-8$ & & & & & $\mathrm{X}$ & \\
$6-10$ & & & & & & $\mathrm{X}$ \\
\hline
\end{tabular}

Table 13 2004-2005: Are different total clashes significantly different?

\begin{tabular}{lllllll}
\hline Clashes & $10-10$ & $10-8$ & $10-6$ & $8-10$ & $8-8$ & $6-10$ \\
\hline $10-10$ & $\mathrm{X}$ & Yes & & & & \\
$10-8$ & & $\mathrm{X}$ & & & & \\
$10-6$ & & & $\mathrm{X}$ & & & \\
$8-10$ & & & & $\mathrm{X}$ & $\mathrm{X}$ & \\
$8-8$ & & & & & & $\mathrm{X}$ \\
$6-10$ & & & & & & \\
\hline
\end{tabular}




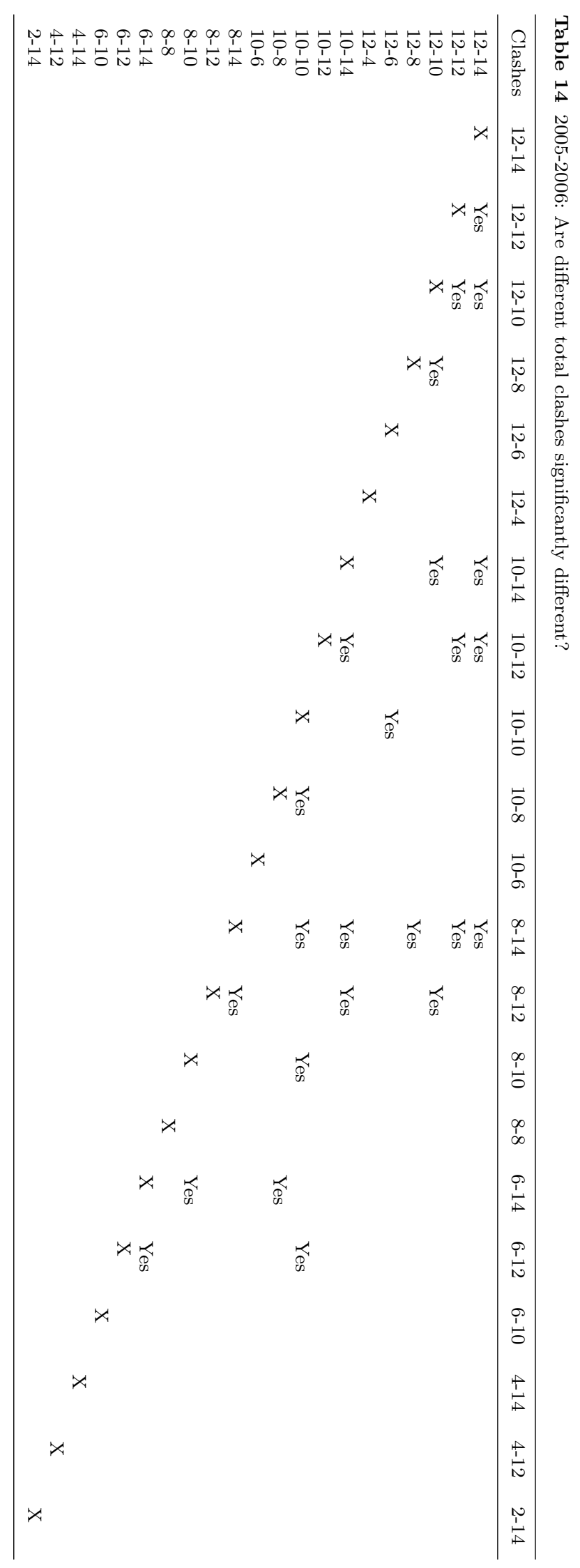


Table 15 2006-2007: Are different total clashes significantly different?

\begin{tabular}{lllllllllll}
\hline Clashes & $14-8$ & $14-6$ & $14-4$ & $14-2$ & $12-8$ & $12-6$ & $12-4$ & $10-8$ & $10-6$ & $8-8$ \\
\hline $14-8$ & $\mathrm{X}$ & Yes & & & Yes & Yes & & Yes & & \\
$14-6$ & & $\mathrm{X}$ & & & & Yes & & Yes & & \\
$14-4$ & & & $\mathrm{X}$ & & & & & & & \\
$14-2$ & & & & $\mathrm{X}$ & & & & & & \\
$12-8$ & & & & & $\mathrm{X}$ & & & Yes & & \\
$12-6$ & & & & & & $\mathrm{X}$ & & & & \\
$12-4$ & & & & & & & $\mathrm{X}$ & & & \\
$10-8$ & & & & & & & & $\mathrm{X}$ & & \\
$10-6$ & & & & & & & & & $\mathrm{X}$ & \\
$8-8$ & & & & & & & & & & $\mathrm{X}$ \\
\hline
\end{tabular}

Table 16 2007-2008: Are different total clashes significantly different?

\begin{tabular}{|c|c|c|c|c|c|c|c|c|c|c|c|c|c|c|c|}
\hline Clashes & $14-10$ & $14-8$ & $14-6$ & $14-4$ & $14-2$ & $12-10$ & $12-8$ & $12-6$ & $12-4$ & $10-10$ & $10-8$ & $10-6$ & $8-10$ & $8-8$ & $6-10$ \\
\hline $14-10$ & $\mathrm{X}$ & & & & & & & & & & & & & & \\
\hline $14-8$ & & $\mathrm{X}$ & & & & & & & & Yes & & & & & \\
\hline $14-6$ & & & $\mathrm{X}$ & & & Yes & & & & & Yes & & Yes & & \\
\hline $14-4$ & & & & $\mathrm{X}$ & & & & & & & & & & & \\
\hline $14-2$ & & & & & $\mathrm{X}$ & & & & & & & & & & \\
\hline $12-10$ & & & & & & $\mathrm{X}$ & Yes & & & Yes & & & & & \\
\hline $12-8$ & & & & & & & $\mathrm{X}$ & & & & Yes & & & & \\
\hline $12-6$ & & & & & & & & $\mathrm{X}$ & & & & & & & \\
\hline $12-4$ & & & & & & & & & $\mathrm{X}$ & & & & & & \\
\hline $10-10$ & & & & & & & & & & $\mathrm{X}$ & & & & & \\
\hline $10-8$ & & & & & & & & & & & $\mathrm{X}$ & & & & \\
\hline $10-6$ & & & & & & & & & & & & $\mathrm{X}$ & & & \\
\hline $8-10$ & & & & & & & & & & & & & $\mathrm{X}$ & & \\
\hline $8-8$ & & & & & & & & & & & & & & $\mathrm{X}$ & \\
\hline $6-10$ & & & & & & & & & & & & & & & $\mathrm{X}$ \\
\hline
\end{tabular}

Table 17 2008-2009: Are different total clashes significantly different?

\begin{tabular}{lllllll}
\hline Clashes & $10-10$ & $10-8$ & $10-6$ & $8-10$ & $8-8$ & $6-10$ \\
\hline $10-10$ & $\mathrm{X}$ & Yes & & Yes & & \\
$10-8$ & & $\mathrm{X}$ & & & & \\
$10-6$ & & & $\mathrm{X}$ & & & \\
$8-10$ & & & & $\mathrm{X}$ & & \\
$8-8$ & & & & & $\mathrm{X}$ & \\
$6-10$ & & & & & & \\
\hline
\end{tabular}

\section{Conclusion}

We have demonstrated that it is sometimes possible to reduce the number of pair clashes without a statistical difference to the distance that has to be travelled by the 
club/supporters. This provides the police with the ability to reduce their costs for these two days, which might have included paying overtime. We hope that we are able to discuss these results with the football authorities and the police in order for them to validate our work and to provide us with potential future research directions. We already recognise that some pair clashes might provide the police with more problems than others and it might be worth prioritising certain clashes so that these can be removed, rather than removing less high profile fixtures. As a longer term research aim, we would like to include in our model details about public transport as some routes might be more difficult than other routes, even if they are shorter. We also plan to run our algorithms for every future season, as well as for previous seasons. Executing the algorithm is not the main issue. Data collection provides the real challenge due to the distance data that has to be collected. To date, this has been carried out manually by using motoring organisation's web sites but we have recently started experimenting with services such as Google Maps ${ }^{\mathrm{TM}}$ and Multimap which will speed up the data collection.

\section{References}

1. Aarts, E., Korst, J., Michels, W.: Simulated annealing. In: E.K. Burke, G. Kendall (eds.) Search Methodologies: Introductory Tutorials in Optimization and Decision Support Methodologies, 1st edn., chap. 7, pp. 97-125. Springer (2005)

2. Anagnostopoulos, A., Michel, L., Van Hentenryck, P., Vergados, Y.: A simulated annealing approach to the traveling tournament problem. Journal of Scheduling 9, 177-193 (2006)

3. Ball, B.C., Webster, D.B.: Optimal scheduling for even-numbered team athletic conferences. AIIE Transactions 9, 161-169 (1977)

4. Bean, J.C., Birge, J.R.: Reducing travelling costs and player fatigue in the national basketball association. Interfaces 10, 98-102 (1980)

5. Cain, W.O.: The computer-aided heuristic approach used to schedule the major league baseball clubs. In: S.P. Ladany, R.E. Machol (eds.) Optimal Strategies in Sports, pp. 33-41. North Holland, Amsterdam (1977)

6. Campbell, R.T., Chen, D.S.: A minimum distance basketball scheduling problem. In: R.E. Machol, S.P. Ladany, D.G. Morrison (eds.) Management Science in Sports, Studies in the Management Sciences, vol. 4, pp. 15-25. North-Holland, Amsterdam (1976)

7. Costa, D.: An evolutionary tabu search algorithm and the NHL scheduling problem. INFOR 33, 161-178 (1995)

8. Di Gaspero, L., Schaerf, A.: A composite-neighborhood tabu search approach to the traveling tournament problem. Journal of Heuristics 13, 189-207 (2007)

9. Dinitz, J.H., Fronček, D., Lamken, E.R., Wallis, W.D.: Scheduling a tournament. In: C.J. Colbourn, J.H. Dinitz (eds.) Handbook of Combinatorial Designs, 2nd edn., pp. 591-606. CRC Press (2006)

10. Drexl, A., Knust, S.: Sports league scheduling: Graph- and resource-based models. Omega 35, 465-471 (2007)

11. Easton, K., Nemhauser, G.L., Trick, M.A.: The travelling tournament problem: Description and benchmarks. In: T. Walsh (ed.) Principles and Practice of Constraint Programming, Lecture Notes in Computer Science, vol. 2239, pp. 580-585. Springer (2001)

12. Easton, K., Nemhauser, G.L., Trick, M.A.: Solving the travelling tournament problem: A combined integer programming and constraint programming approach. In: E. Burke, P. de Causmaecker (eds.) The 4th International Conference on the Practice and Theory of Automated Timetabling, Lecture Notes in Computer Science, vol. 2740, pp. 100-109. Springer (2003)

13. Easton, K., Nemhauser, G.L., Trick, M.A.: Sports scheduling. In: J.T. Leung (ed.) Handbook of Scheduling, pp. 52.1-52.19. CRC Press (2004)

14. Elf, M., Jnger, M., Rinaldi, G.: Minimizing breaks by maximizing cuts. Operations Research Letters 31(3), 343-349 (2003)

15. Evans, J.R.: A microcomputer-based decision support system for scheduling umpires in the American Baseball League. Interfaces 18, 42-51 (1988) 
16. Ferland, J.A., Fleurent, C.: Computer aided scheduling for a sport league. INFOR 29, $14-25$ (1991)

17. Kendall, G.: Scheduling English football fixtures over holiday periods. Journal of the Operational Research Society 59, 743-755 (2008)

18. Kendall, G., Knust, S., Ribeiro, C.C., Urrutia, S.: Scheduling in sports: An annotated bibliography. Computers \& Operations Research 37, 1-19 (2010)

19. Kendall, G., While, L., McCollum, B., Cruz, F.: A multiobjective approach for UK football scheduling. In: E.K. Burke, M. Gendreau (eds.) Proceedings of the 7th International Conference on the Practice and Theory of Automated Timetabling (2008)

20. Knust, S.: Classification of literature on sports scheduling (2010). Available online at http://www.inf.uos.de/knust/sportssched/sportlit_class/, last visited 15th July 2010

21. Rasmussen, R.V., Trick, M.A.: Round robin scheduling - A survey. European Journal of Operational Research 188, 617-636 (2008)

22. Ribeiro, C.C., Urrutia, S.: Heuristics for the mirrored traveling tournament problem. European Journal of Operational Research 179, 775-787 (2007)

23. Trick, M.: Traveling tournament problem instances (2010). Available online at http://mat.gsia.cmu.edu/TOURN/, last accessed 15th July 2010

24. Urrutia, S., Ribeiro, C.: Minimizing travels by maximizing breaks in round robin tournament schedules. Electronic Notes in Discrete Mathematics 18-C, 227-233 (2004)

25. Urrutia, S., Ribeiro, C.C., Melo, R.A.: A new lower bound to the traveling tournament problem. In: Proceedings of the IEEE Symposium on Computational Intelligence in Scheduling, pp. 15-18. IEEE, Honolulu (2007)

26. de Werra, D.: Scheduling in sports. In: P. Hansen (ed.) Studies on Graphs and Discrete Programming, pp. 381-395. North Holland, Amsterdam (1981)

27. de Werra, D.: Some models of graphs for scheduling sports competitions. Discrete Applied Mathematics 21, 47-65 (1988)

28. Wright, M.: Timetabling county cricket fixtures using a form of tabu search. Journal of the Operational Research Society 45, 758-770 (1994)

29. Wright, M.: 50 years of OR in sport. Journal of the Operational Research Society 60, S161-S168 (2009) 